\title{
Automated System for Detection of High Risk Diabetic Macular Edema in Color Retinal Images
}

\author{
Anaswara Chandran \\ Assistant Professor \\ Department of Computer \\ Science and Engineering \\ Karpagam Institute of \\ Technology, Coimbatore
}

\author{
Nisha K. K. \\ Assistant Professor \\ Department of Computer \\ Science and Engineering \\ R.I.T Kottayam, India
}

\author{
Laxmi Raja \\ Assistant Professor \\ Department of Computer \\ Science and Engineering \\ Karpagam Institute of \\ Technology, Coimbatore
}

\begin{abstract}
The detection of macula and classification of macular edema condition is an open issue for researchers in the field of ophthalmology. Diabetic Macular Edema (DME) is a secondary disease associated with DR (Diabetic Retinopathy), and it acts as a major reason for vision loss in diabetic patients. In this paper an automated system is presented with a Binarized Macula Detection (BMD) algorithm for detection of macula, irrespective of the location of Optic Disk (OD). The work tries to target high risk DME which occurs within 1 Optic Disk Diameter (ODD) area in the macular region. This will help to provide immediate treatment to the patients with high risk of DME. The extraction of texture features from the green channel of Region of Interest (ROI) reduces the time for working with whole image, and the classification system with Support Vector Machine (SVM), K Nearest Neighbor (KNN) and Random Forest classifiers predicts the individual result for input images. The binary classification of Stage 0 (Normal) and Stage 2 (High Risk Diabetic Macular Edema) was performed on dataset from MESSIDOR database, and the SVM classifier with RBF kernel obtained highest accuracy and specificity as $94.58 \%$ and $96.94 \%$ respectively. Higher sensitivity was obtained by KNN as $89.66 \%$. The system was also compared with a feature reduction system using Principal Component Analysis (PCA) and the system with, SVM and RBF kernel without feature reduction technique outperformed the results.
\end{abstract}

\section{Keywords}

Diabetic Retinopathy; Diabetic Macular Edema; Exudates; High Risk DME; BMD Algorithm; Principal Component Analysis.

\section{INTRODUCTION}

Diabetes Mellitus is an infirmity caused by the imbalance of insulin hormone in human body. In the absence of insulin, the body fails to use glucose for energy mechanism. As a result the blood glucose level increases and causes damage to blood vessels in many organs like Kidney, Heart, Eyes and Nervous System. Diabetic eye diseases include a group of eye disorders that affect people with diabetes. They include DR, DME, cataract and glaucoma. All these diabetic eye diseases have the potential to affect normal vision. The diabetic retinopathy is referred to the condition where the high blood glucose level affects the retinal blood vessels of human eye. The changes in the retinal blood vessel will cause them to bleed or leak fluid, affecting the vision. The leakage of blood and fluid will create the structures like exudates in retina.
DME is a secondary disease of diabetic retinopathy condition, which is characterized by swelling in the macular region due to the fluid leakage. The fluid leakage will generate structures like soft and hard exudates in the macular region of retina. Macula is the portion of the retina responsible for straight ahead vision that helps in reading, recognizing faces, driving and central vision. Macula holds tightly packed cones, which provide sharp, clear, detailed central vision that enables to see in detail the form and color that is directly in the centre of the field of view. The DME is the most common cause for vision loss for people with diabetic retinopathy.

The DME is referred to as high risk diabetic macular edema, when the exudates are present within 1 ODD of the macular region. This advanced stage requires immediate treatment and can lead to severe vision impairments. Figure 1 shows retinal images with macula and optic disk labels and Stage 0 and Stage 2 images from MESSIDOR database.

The increased number of patients acts as a difficult task for the ophthalmologists to perform diagnosis. The ratio of normal to abnormal diabetic retinopathy patients is about 9 to 1 [1]. The efficient computer aided systems can contribute more to this problem and can help in the screening programs of diabetic macular edema. The challenge in computer aided systems lies in correct detection of anatomical structures from the color retinal images. The diabetic macular edema detection systems require analyzing the macular region for the presence of abnormal structures like exudates.

A survey was conducted among the ophthalmologists to gather the information about the current detection systems used for macular edema detection. The frequently reported techniques for high risk macular edema detection are Fundus Fluroscein Angiography (FFA) and Optical Coherence Tomography (OCT). The results revealed the fact that, the techniques such as FFA causes side effects on hypertensive patients with renal diseases due to the use of fluroscein dye. The OCT evaluation also result increase in blood pressure and adverse reactions to dilating drops in several cases. This shows that the automated systems can contribute more to the field.

Number of literature is available on the macula detection methods and macular edema identification systems. K. Sai Deepak et al. [2] proposed a method to classify the fundus images in to normal and DME based on the global characteristics of image. Sreejini K. S. et al. [3] proposed an unsupervised detection system to detect the severity of the DME. The macula was detected with respect to the optic disk in the retinal image using circular binary masks. Aditya 

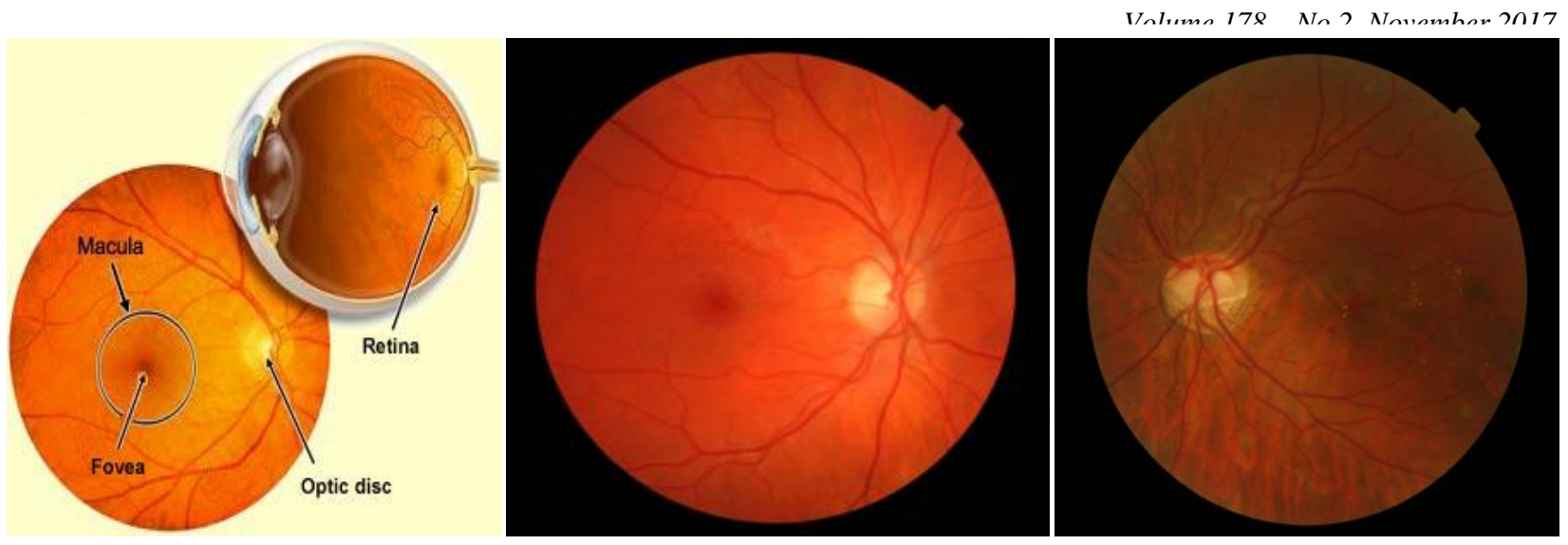

Figure 1: a) Retinal image with marked macula and Optic Disk b) Stage 0, Normal Image c) Stage 2, Abnormal Image

Kunwar et al. [4] proposed a system for detection of high risk macular edema using texture features and SVM classifier. The centre of the optic disk was found using [5]. The classification was based on region of interest extracted, and it reduced the complexity of processing the whole image.

In most of the literatures the macula detection was based on the optic disk, which can cause misclassification in case of blurred optic disk. The aim of this paper is,

1. To generate an algorithm for macula detection irrespective of the position of optic disc

2. Provide a binary classification of Stage 0 and Stage 2 for high risk DME detection

3. Provide a comparative study for assessing the best matching classifier for the high risk DME detection.

4. Compare the result of classifier based on PCA feature reduction system

The classifiers Random Forest, KNN and SVM are used here for making individual decision on input image. The paper focuses on the Stage 0 and Stage 2 cases of DME, since the stage 2 case shows high risk of DME. Thus the macula detection algorithm proposed here marks the 1ODD around the macula as the ROI for the system. High risk DME detection system extracts texture features from the ROI and performs classification.

This paper is distributed as follows, section II contains the related works in the field and section III describes the proposed methodology like image pre-processing, BMD Algorithm, Feature Extraction and Classification. The results and discussions are included in section IV followed by an overall conclusion in the section $\mathrm{V}$.

\section{RELATED WORKS}

Various methods are available, including techniques for detection of macula from retinal images and classification of images based on the DME severity. These automated systems can improve the efficiency of eye-health care services.

Liu et al. [6] proposed an automatic retinal image analysis system which was useful for the mass screening of diabetic retinopathy. The fundus photographs were processed to identify the optic disk and the fovea through hough transform. The authors mentioned that the evaluation was performed on images obtained from local hospital in Singapore, and the system was failed to identify the macular region from few cases with blurred optic disk. In order to address this higher level addressing of structural information from the optic disk is required.

The methodology described by Liu et al. [6] failed to provide better results when the optic disc is blurred and when large exudates with same features as optic disc are present. Optic disc identification based on the geometrical relationship between the optic disc and fovea is also impractical due to the complex task of vessel detection. Sagar et al. [7] developed a system for automatic detection of the OD and macula in the eye retinal fundus images. The green plane was used due to the greater distribution of intensity through the image. Optic disc localization is done by iterative threshold method to detect initial set of candidate regions and then connected component analysis is performed to locate the actual optic disc. The fovea in the retinal image is identified by finding the darkest region. This will be followed by the priori geometric criteria based on anatomy of human eye. The proposed work for identifying the anatomical structures provides better results except for the cases with presence of large lesions around the optic disc.

Tan et al introduced in [8] an automatic method for macula detection in the retinal fundus image. The entire working of the system starts with classifying the retinal image as the right or left eye followed by the creation of circular mask. Using the argali program the coordinates of centre of the disc and height of the disc were calculated. The masked image was then converted into the gray image and region of interest is selected. This method demonstrates better results on evaluation with publically available datasets. The system moderates the chance of detecting blood vessels, which are darker. The limitation of this method include sometimes the boundary of the circle mask will not cover the macula which leads to false detection. The future modification proposed was instead of classifying the images as left and right, the macula can be identified with the relative position of the optic disc.

Umer et al. [9] proposed an automated system for early detection of macular edema to identify all possible exudates present in the retinal surface. The system consists of three stages including candidate exudates detection, feature extraction and classification. Gabor filter bank was used for the detection of bright lesions in the image. Gabor filter is used for its ability to work with different orientations. The candidate exudates detection will give set of possible regions that can be exudates, and the features are selected from the region. 
Deepak et al. [2] proposed and evaluated a method for diabetic macular edema detection and assessment. The work provides a hierarchical approach to the problem, for classification of image and prediction of severity. The methodology enhances the existing DR screening infrastructure for DME detection. They have resized the image, and then detected the optic disc by using intensity in red channel. Macula is detected with reference to the OD position. This method has a high chance of wrong identification of optic disc as exudates under noise and reflections. To overcome this difficulty in works involving the macula identification with respect to optic disc, a region based method which is independent of optic disc location is prescribed by Sengar et al. [10]. After the region based detection of macula, the morphological dilation operator is used to detect the hard exudates present in the area. Grading of DME was done on the basis of region wise information of exudates. The unwanted artifacts like blood vessels and optic disc are removed from the image and based on the information from the image regions; the severity was predicted for the diabetic macular edema cases. The region wise information extraction can act as a base for other literature works but the exudates detection can incorporate with several features since the paper conveys only about the morphological dilation operator.

Rodrigo Veras, Fatima Medeiros et al. [11] proposed a work that can be useful to assess the accuracy of macula detection algorithms on public database. The comparison among four macula detection methods were provided based on the set of rules generated. The work can act as a base for any researcher and specialists in ophthalmology to assess the techniques and develop DME detection systems. The literatures described above show several limitations with the macula detection systems, based on optic disk. In cases of blurred optic disks there are chances for false detections. The proposed methodology attempts a macula detection system working irrespective of the optic disk, and a classification system for differentiating high risk DME and normal cases.

\section{METHODOLOGY}

The architecture of the High Risk Macular Edema Detection System is shown in Figure 2. It starts with image acquisition, image preprocessing followed by macula detection using BMD algorithm, feature extraction, classification and performance evaluation. The core part of any macular edema detection system is the macular region identification from color retinal images. BMD algorithm is used for detection of macular region from color retinal images obtained from the MESSIDOR database. The fovea part is identified and the ROI around the fovea is selected as the macular region. The texture features for the Stage 0 and stage 2 images will vary, and the classifiers Random forest, SVM and KNN are used for predicting the individual results. Performance evaluation is made to identify the best performing classifier in the scenario.

\subsection{Image Acquisition}

Images are obtained from the publically available MESSIDOR [12] database. The MESSIDOR database was formed to promote studies on computer aided diagnoses of diabetic retinopathy and secondary diseases. The 1200 eye fundus color images of the MESSIDOR database were acquired by 3 ophthalmologic departments using a color video 3CCD camera on a Topcon TRC NW6 non-mydriatic radiograph with a 45 degree field of view. Two diagnoses have been provided by the medical experts for each image:
Retinopathy grade and Risk of macular edema. The macular edema diagnosis is based on Early Treatment Diabetic Retinopathy Studies (ETRDS) grading for DME. It shows three stages Stage 0: Normal, Stage 1: Shortest distance between macula and hard exudates is more than $1 \mathrm{DD}$ from fovea and Stage 2: Shortest distance between macula and hard exudates is less than or equal to $1 \mathrm{DD}$ from fovea. For the high risk DME detection system the severity scales Stage 0 and Stage 2 are chosen.

\subsection{Image Preprocessing}

The input retinal image is pre processed before the macula detection stage. The color retinal image is converted to the gray scale image here. The gray scales of the image contain maximum intensity values of corresponding pixels, and are technically useful in medical imaging and remote sensing problems. The image contains 256 gray levels, where 0 shows black and the maximum value is 255 .

\subsubsection{Binarized Macula Detection (BMD)} Algorithm

The BMD algorithm takes the gray scale image as input. Reconfilt function is used to segment circular locally darker regions in the grayscale image, using a circular kernel and threshold sensitivity. The minimum and maximum radius is also passed as the required input parameters. The binary image generated by reconfilt function is subjected to next level of screening in terms of upper bound and lower bound area limits. The structural elements with disk shape are filtered out, and morphological operations are performed to fill and close the binary image. Region properties of the remaining blobs are calculated and a final screening based on a metric threshold is performed on the binary image. The threshold value was set to be as 0.4 and the metric was calculated using area and perimeter.

The structures with metric value greater than threshold are arranged in the descending order of their areas, and the first structure in the sorted index is taken as the macular region. The centroid of the structure is plotted on the color retinal image and it is marked as the fovea of the retinal image. The immediate 1 ODD around the fovea is responsible for high risk macular edema condition. From [13], 1 ODD nearly equals to 276 pixels in Messidor datasets. Thus macular region around the fovea with radius 138 pixels is marked as the ROI and is extracted from the retinal image. Figure 3 shows various stages in BMD algorithm.

\subsubsection{Algorithm 1: BMD \\ ip: Gray Scale Image \\ op: Region of Interest in macula \\ $\mathrm{I}=$ reconfilt (gray Image, threshold, minimum radius, \\ Select structural elements with disk shape \\ Morphological imclose \& imfill operations \\ Find regionprops (I, 'Area', 'Centroid') for k objects; \\ Set threshold and find area \& perimeter}

$$
\text { Use metric }=4 \pi \text { arear } / \text { Perimeter }^{2}
$$

if metric > threshold

find centroid and area

end

if $\mathrm{k} \sim=1$

sort areas in descending order

select first index 
plot centroid (fovea)

end

maximum radius);
Set lower bound \& Upper bound areas on binary image I Select Region of Interest (1 ODD from fovea)

Extract Region of Interest (1 ODD from fovea)

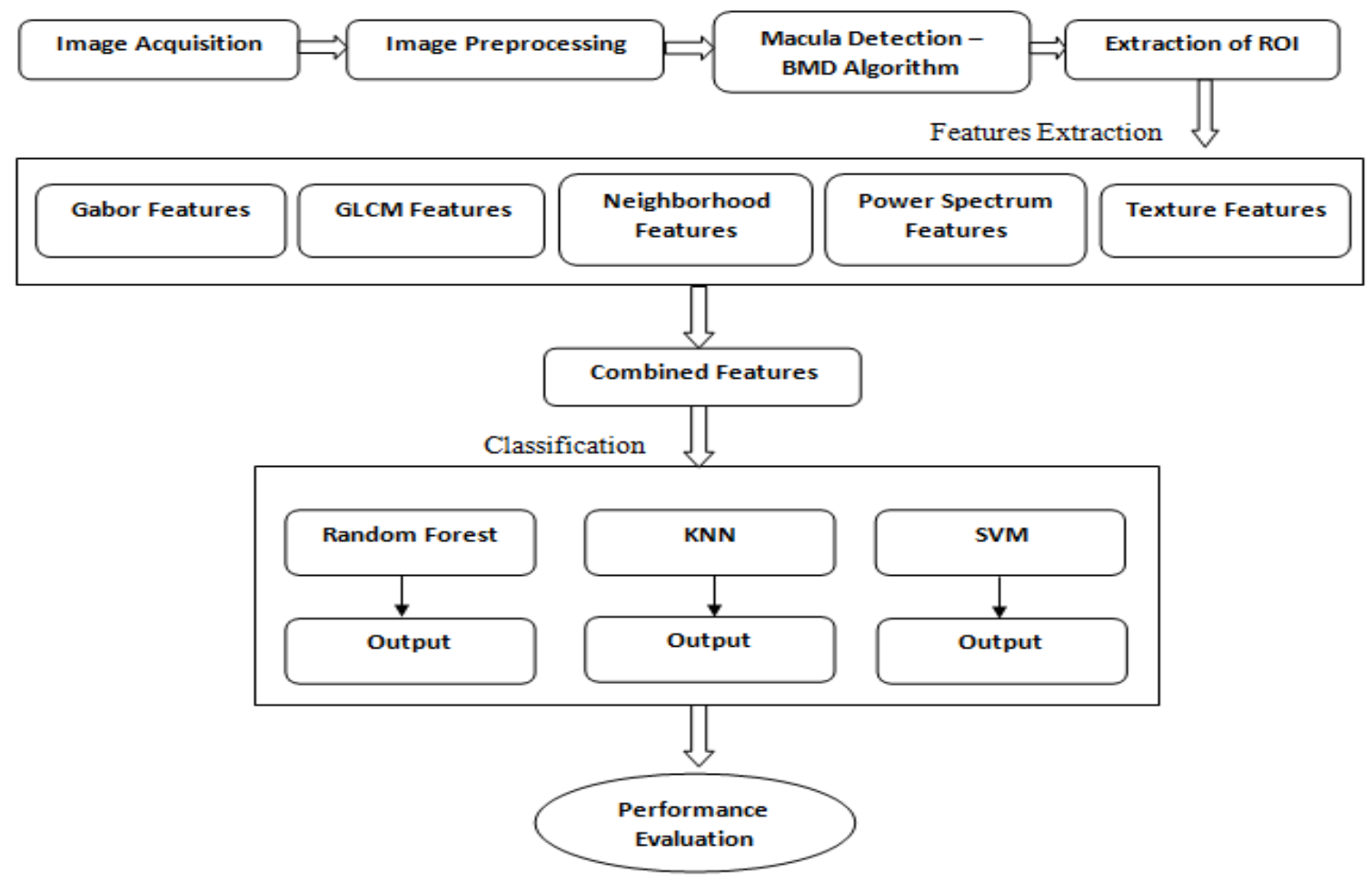

Figure 2: High Risk Diabetic Macular Edema Detection System

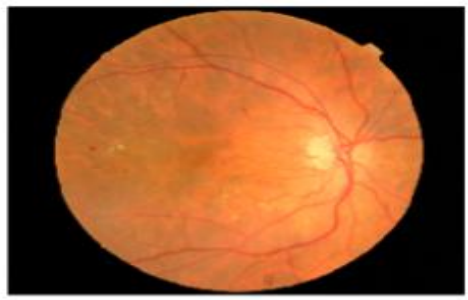

a)

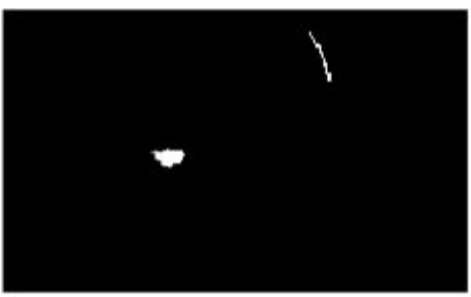

d)

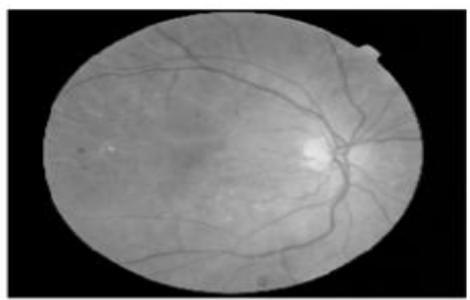

b)

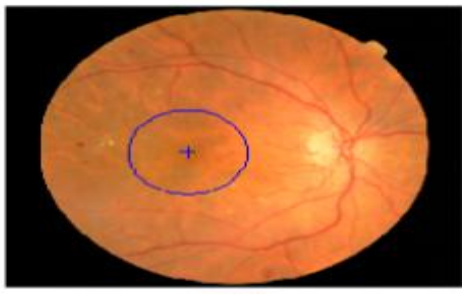

e)

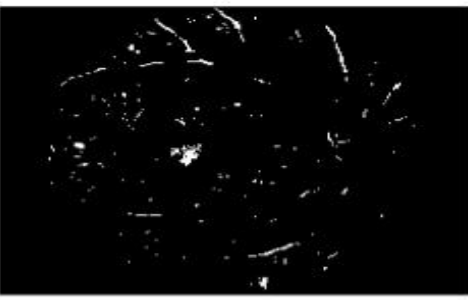

c)

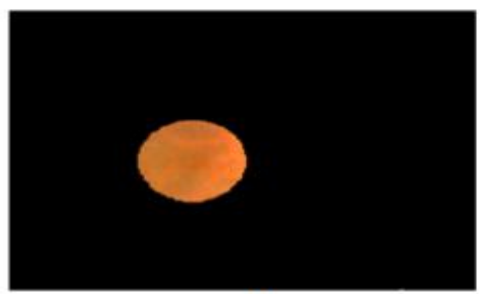

f)
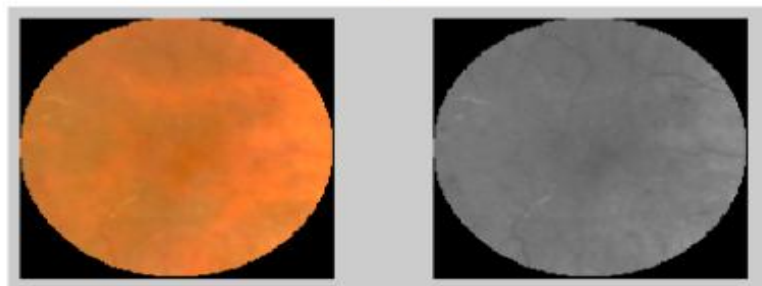

Figure 3: a) Input Retinal Image. b) Gray Image, c) \& d) Processing of BMD Algorithm, e) \& f) ROI in input image, g) Cropped ROI and Green channel of ROI 


\subsection{Feature Extraction}

Gabor filter is a linear filter used for edge detection. Its impulse response is defined as the product of sinusoidal wave and gaussian function. Using log gabor, response of green channel of the ROI is fed in to a gabor filter bank. The benefit of using log gabor over original gabor filter is that, it better fits the statistics of natural images. Number of orientations and scales used are 12 and 4 respectively. The mean of 2 nd 5 th moments of 12 orientations and 4 scales are taken as $\log$ gabor responses. 48 features are obtained from the gabor filter responses from the ROI.

The features from Gray Level Co occurrence Matrix (GLCM) are extracted using 32 level graycomatrix in 4 orientations $(0$, $45,90,135$ degrees). Graycomatrix analyze pairs of horizontally adjacent pixels in a scaled version of image. It accumulates the total occurrence of each combination, producing an output array, GLCMS. Contrast, correlation, energy and homogeneity features from a 32 level cooccurrence matrix of green channel of the ROI are extracted.

Neighborhood features include mean and variance of range filter and entropy filter response. Range filter calculates the local range of an image, where the local entropy of the grayscale image is calculated using entropy filter. Power Spectrum Density Features are calculated as the integral of fourier power spectral density in 7 annular frequency bands are calculated.

From the ROI the basic texture features such as mean, standard deviation, variance and entropy are calculated. The entire features calculated above are combined together to form 79 feature set, on the basis of which the classification performs.

\subsection{Classification}

The classifiers Random Forest, KNN and SVM are used to predict the individual decision on the input color retinal image.

Random forest classifier is an ensemble method for classification. It constructs magnitude of decision trees at training time and output the class that is mode of the classes or mean prediction of the individual trees. The idea is to create an RF predictor that discriminate the test data from the suitably generated synthetic data. Breiman and Cutler's Random Forest Package [14] is used to perform classification. The classRF_train is used to create model for the classifier with input parameters trained feature set and trained labels. The number of trees is set as 1000 . Once the model is generated the class RF_predict outputs the obtained label for the test image based on combined features and the model.

A KDTreeSearcher object performs supervised KNN classification using a kd-tree. Kd-trees divide trained feature sets into nodes with at most BucketSize (default is 50) points per node. When prediction on input image is required, the KNN search determines the nodes to which the combined features belong. It finds the closest $\mathrm{k}$ points within that node and its distance to the combined features. It chooses all other nodes having any area that is within the same distance in any direction. Nodes within the range for any points closer to the combined features are also searched, and return the predicted label for input image.

SVM classifier is used in binary classification for the prediction of stage 0 or stage 2 labels for input image. SVM classifies data by finding the best hyper plane that separates all data points of different classes. The finest hyper plane for an SVM is the one with the largest margin between the two classes, and the data points that are closest to the hyper plane are called as support vectors. SVM classifier uses structure
SVMSTRUCT, created using SVMTRAIN, and returns the predicted class label for input. The SVM classifier uses kernel functions to map the training data to the kernel spaces. The different kernel functions used in SVM include, Linear, Quadratic, Polynomial, RBF (Radial Basis Function) and MLP (Multilayer Perceptron). The default kernel is linear. The high risk DME detection system is checked with different kernel functions of the SVM classifier to check the best fit kernel.

\subsection{Performance Evaluation}

The purpose of evaluation in the high risk DME detection system is to select the best classifier to detect the abnormal and normal images. The classifiers Random forest, SVM and $\mathrm{KNN}$ are trained with same set of images from the MESSIDOR database. On each input the classifiers predicts the set of labels and are compared with the ground truth of the images. The test image set selected from MESSIDOR was used to evaluate the performance of classifiers on more number of same images. The accuracy of SVM was also evaluated for different kernels. The section IV shows the detailed results and discussions.

\section{RESULTS AND DISCUSSION 4.1 BMD Algorithm}

From MESSIDOR database total 651 images were selected which include 504 Stage 0 images and 147 Stage 2 images. BMD algorithm was executed on dataset and the ROI was extracted as 1ODD area around the fovea. Out of 651 input images the macula was correctly detected in 547 retinal images. The BMD algorithm identified the macula in retinal images with an accuracy of $84.03 \%$. Thus algorithm shows satisfactory results, and reduced the overhead of identification of the optic disk for macula detection. In none of the images the optic disk was marked as fovea, which implies that the presence of OD in retinal image will not affect the identification of macula, thus eliminating the procedures OD identification, masking and left/right eye image classification.

\subsection{Classification System}

The train set with 326 images was used to train the classifiers and test set of 221 was used for the evaluation of the classifiers. From green channel of the ROI extracted using the BMD algorithm a total of 79 texture features were extracted. Using the trained feature sets and the trained label, classifiers Random Forest, SVM and KNN predicted the outcome for the input images.

The result of SVM was evaluated on different kernels and the RBF kernel found to outperform the rest. The kernel functions polynomial and quadratic failed to achieve convergence within maximum number of iterations.

The result of classification system was compared with a system using PCA (Principal Component Analysis) for feature reduction. The PCA is used for reducing the dimensionality of the feature space. The concept is to generate the principal components, which have associated eigen value representing the standard deviation of the sample. The least influential features can be eliminated to reduce the feature space. The number of principal components are reduced to 20 and $99 \%$ of the variance is retained. The system was tested with same test and train set. The classification result of high risk DME detection system and PCA based system, including different SVM kernels in this case are shown in table 1.

The entire system consumes 104 seconds for execution in mat lab R2013. The computation was performed on a system with 
configuration Intel Celeron processor with $2 \mathrm{~GB}$ amount of RAM. The function classperf in matlab was used to measure the performance measures; accuracy, sensitivity and specificity. The high risk DME detection system shows high accuracy of $94.58 \%$ and specificity of $96.94 \%$ using SVM classifier with RBF kernel. Higher sensitivity that is the ability to detect Stage 2 images from Stage 0 is shown by KNN as $89.66 \%$. SVM with different kernels shows comparatively lower performance, on using PCA for feature reduction. The classifiers KNN and Random Forest shows high performance measures than SVM on PCA based system. The ROC curve for the high risk DME classification system is shown in Figure 4, and area under curve is higher for SVM (RBF).

Table 1. Classification Result

\begin{tabular}{|c|c|c|c|c|c|c|c|}
\hline \multicolumn{8}{|c|}{ High Risk DME Detection System } \\
\hline $\begin{array}{l}\text { Classi } \\
\text { fier }\end{array}$ & TP & $\mathbf{T N}$ & FP & $\begin{array}{l}\mathbf{F} \\
\mathbf{N}\end{array}$ & $\begin{array}{c}\text { Acc } \\
\text { urac } \\
\mathbf{y} \\
(\%)\end{array}$ & $\begin{array}{c}\text { Sens } \\
\text { itivi } \\
\text { ty } \\
(\%)\end{array}$ & $\begin{array}{l}\text { Specific } \\
\text { ity }(\%)\end{array}$ \\
\hline RF & 51 & 133 & 30 & 7 & $\begin{array}{c}83.2 \\
6\end{array}$ & $\begin{array}{c}87.9 \\
4\end{array}$ & 81.6 \\
\hline KNN & 52 & 112 & 51 & 6 & $\begin{array}{c}74.2 \\
1\end{array}$ & $\begin{array}{c}89.6 \\
6\end{array}$ & 68.72 \\
\hline $\begin{array}{l}\text { SVM } \\
\text { RBF }\end{array}$ & 51 & 158 & 5 & 7 & $\begin{array}{c}94.5 \\
8\end{array}$ & $\begin{array}{c}87.9 \\
4\end{array}$ & 96.94 \\
\hline $\begin{array}{l}\text { SVM } \\
\text { Linea } \\
\text { r }\end{array}$ & 48 & 104 & 59 & 10 & $\begin{array}{c}68.7 \\
8\end{array}$ & $\begin{array}{c}82.7 \\
6\end{array}$ & 63.81 \\
\hline $\begin{array}{l}\text { SVM } \\
\text { MLP }\end{array}$ & 58 & 0 & 163 & 0 & $\begin{array}{c}26.2 \\
5\end{array}$ & 100 & 0 \\
\hline \multicolumn{8}{|c|}{ PCA Based System } \\
\hline RF & 53 & $\begin{array}{c}12 \\
1\end{array}$ & 42 & 5 & $\begin{array}{c}78.7 \\
4\end{array}$ & $\begin{array}{c}91.3 \\
8\end{array}$ & 74.24 \\
\hline KNN & 53 & $\begin{array}{c}12 \\
1\end{array}$ & 42 & 5 & $\begin{array}{c}78.7 \\
4\end{array}$ & $\begin{array}{c}91.3 \\
8\end{array}$ & 74.24 \\
\hline $\begin{array}{l}\text { SVM } \\
\text { RBF }\end{array}$ & 23 & 97 & 66 & 35 & 54.3 & $\begin{array}{c}39.6 \\
6\end{array}$ & 59.51 \\
\hline $\begin{array}{l}\text { SVM } \\
\text { Linear }\end{array}$ & 58 & 0 & 163 & 0 & $\begin{array}{c}26.2 \\
5\end{array}$ & 100 & 0 \\
\hline $\begin{array}{l}\text { SVM } \\
\text { MLP }\end{array}$ & 41 & 57 & 106 & 17 & $\begin{array}{c}44.3 \\
5\end{array}$ & $\begin{array}{c}70.6 \\
9\end{array}$ & 34.97 \\
\hline
\end{tabular}

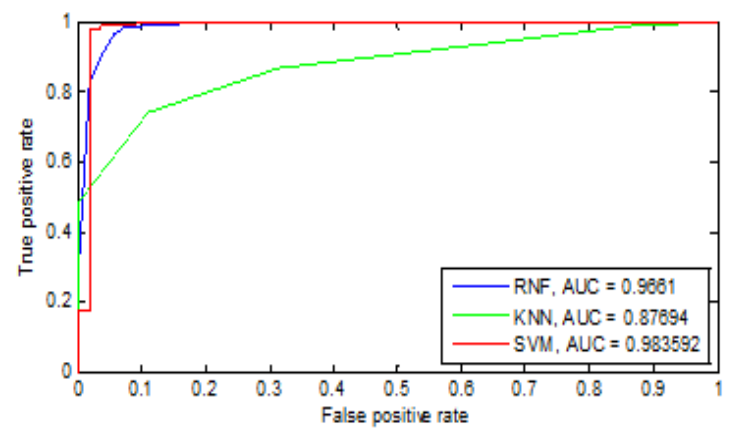

Figure 4: ROC for High Risk DME Classification

\section{CONCLUSION}

The proposed system was designed to provide assistance to the high risk DME detection problem. Apart from the methods working with optic disk localization and macula detection, the work proposed BMD algorithm for the identification of fovea and the detection of ROI thereby. The satisfactory results of BMD algorithm on the MESSIDOR database shows the idea is addressing the problem, and can be enhanced for better results. The future work may include considering more information about bright lesions in other areas of retina, to avoid false predictions of fovea.

The classification was performed, based on the features extracted from the green channel of ROI, and on evaluation of the results, SVM classifier on RBF kernel reports higher accuracy and specificity without the use of feature reduction technique. KNN shows higher sensitivity on the test data which implies the ability to detect high risk DME cases correctly. The comparison of the system with PCA based implementation revealed that the SVM shows comparatively lower results. KNN and Random forest better fits with the The heading of a section should be in Times New Roman 12-point bold in all-capitals flush left with an additional 6-points of white space above the section head. Sections and subsequent sub- sections should be numbered and flush left. For a section head and a subsection head together (such as Section 3 and subsection 3.1), use no additional space above the subsection head.

\section{ACKNOWLEDGMENTS}

The retinal images used in this work were kindly provided by the MESSIDOR program partners (see http://www.adcis.net/en/DownloadThirdParty/Messidor.html).

\section{REFERENCES}

[1] 1. M. D. Abramoff, M. Niemeyer, M. S. SuttorpSchulten, M. A. Viergever, S. R. Russell, and B. van Ginneken, "Evaluation of a system for automatic detection of diabetic retinopathy from color fundus photographs in a large population of patients with diabetes," J. Diabetes Care, vol. 31, no. 2, pp. 193-198, Nov. 2007.

[2] K. Sai Deepak, Jayanthi Sivaswamy, "Automatic assessment of Macular Edema from Color Retinal Images", IEEE Transactions on Medical Imaging, Vol.31, NO. 3, pp. 766-776, March2012.

[3] Sreejini K. S., V.K. Govindan, "Automatic Grading of severity of Diabetic Macular Edema using Color Fundus Images", International Conference on Advances in Computing and Communication,pp. 177-180, 2013.

[4] Aditya Kunwar1,Shrey Magotra,M Partha Sarathi, "Detection of High-Risk Macular Edema using Texture features and Classification using SVM Classifier", 9781-4799-8792-4/15/\$31.00_c 2015 IEEE.

[5] Malay Kishore Dutta, M. Parthasarathi, Shaumik Ganguly, Shaunak Ganguly, Kshitij Srivastava, "An efficient image processing based technique for comprehensive detection and grading of nonproliferative diabetic retinopathy from fundus images", Computer Methods in Biomechanics and Biomedical Engineering: Imaging \& Visualization,July,2015.

[6] Z. Liu, C. Opas, and S. Krishnan. Automatic image analysis of fundus photograph. In EMBS, volume 2, pages 524-525, 1997. 
[7] A. Sagar, S. Balasubramanian, and V. Chandrasekaran. Automatic detection of anatomical structures in digital fundus retinal images. In IAPR - MVA, pages 483-486, 2007.

[8] N. Tan, D. Wong, J. Liu, W. Ng, Z. Zhang, J. Lim, Z. Tan, Y. Tang, H. Li, S. Lu, and T. Wong, "Automatic detection of the macula in the retinal fundus image by detecting regions with low pixel intensity" In ICBPE, pages $1-5,2009$.

[9] Umer Aftab, M. Usman Akram, "Automated Identification of Exudates for Detection of Macular Edema", Cairo International Biomedical Engineering Conference, Cairo, Egypt, pp. 27-30, December2012.

[10] Namita Sengar, Malay Kishore Dutta, Radim Burget, and Lukas Povoda, "Detection of Diabetic Macular Edema in Retinal Images Using a Region Based Method", 978-14799-8498-5/15/\$31.00 @2015 IEEE.
[11] Rodrigo Veras, Fátima Medeiros ; Romuere Silva ; Daniela Ushizima, "Assessing the accuracy of macula detection methods in retinal images", 2013 18th International Conference on Digital Signal Processing (DSP), Fira, 1-6, 1-3 July 2013.

[12] Decenciere et al. Feedback on a publicly distributed database: the Messidor database. Image Analysis \& Stereology, v. 33, n. 3, p. 231-234, aug. 2014. ISSN 1854-5165.

[13] T. Lim, W.M.D.W. Zaki, A. Hussain, S.L. Lim, S. Kusalavan, "Classification of Diabetic Macular Edema in Digital Fundus Images", 2011 IEEE Colloquium on Humanities, Science and Engineering Research (CHUSER 2011), Dec 5-6 2011, Penang.

[14] A. Liaw and M. Wiener (2002), "Classification and Regression by random forest”, R News 2(3), 18-22. 